\title{
Precise test of quantum jump theory
}

\author{
Randall G. Hulet, ${ }^{*}$ D. J. Wineland, J. C. Bergquist, and Wayne M. Itano \\ Time and Frequency Division, National Bureau of Standards, Boulder, Colorado 80303
}

(Received 16 November 1987)

\begin{abstract}
Quantum jumps due solely to spontaneous Raman scattering between the Zeeman sublevels of a single ${ }^{24} \mathrm{Mg}^{+}$ion have been observed in the fluorescence emitted by the ion. A theory of quantum jumps for this system predicts that coherences between excited levels cause the ratio of the mean duration of the "fluorescence-on periods" to the mean duration of the "fluorescence-off periods" to be independent of laser intensity. The measured value agrees with the predicted one to within the measurement precision of $2 \%$. The distribution of the durations of the off periods also agrees with theory.
\end{abstract}

A few groups have recently observed "quantum jumps" between the energy levels of a single atomic ion as signaled by an abrupt change in the fluorescence emitted by the ion. ${ }^{1-3}$ In another experiment, ${ }^{4}$ quantum jumps were inferred from the statistics of the fluorescence emitted by neutral atoms in a weak beam. In these experiments, the quantum jump was a transition (either stimulated or spontaneous) into or out of a metastable excited state.

In this paper, we report the observation of quantum jumps due solely to spontaneous Raman transitions into and out of ground-state sublevels. As opposed to the earlier experiments, we quantitatively compare the predictions of the theory with the experiment. A particular realization of the process ${ }^{5}$ is illustrated in Fig. 1. It employs just one radiation source which is tuned between a pair of Zeeman levels of an atom or ion with a ${ }^{2} S_{1 / 2}$ ground state

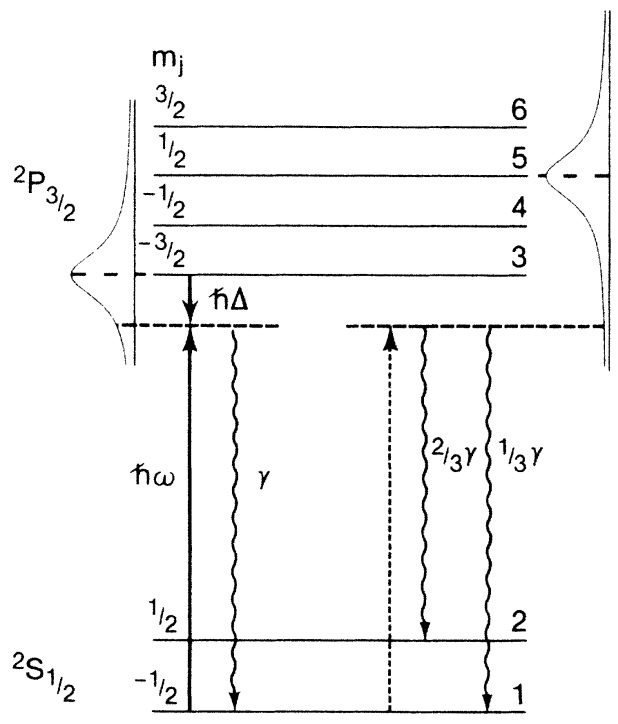

FIG. 1. The energy-level structure of the $S_{1 / 2}$ and $P_{3 / 2}$ states of an atom in a magnetic field (not to scale). A laser of frequency $\omega$ is assumed to be tuned near the $S_{1 / 2}, m_{J}=-\frac{1}{2} \leftrightarrow P_{3 / 2}$, $m_{J}=-\frac{3}{2}$ transition frequency $\omega_{0}$. The energy separation of adjacent $P_{3 / 2}\left(S_{1 / 2}\right)$ sublevels is $\hbar \alpha / 2(3 \hbar \alpha / 4)$. The indicated Lorentzian widths and detuning $\Delta$, which are much less than $\alpha$, have been exaggerated for clarity. and an excited ${ }^{2} P_{3 / 2}$ state (for example, an alkali atom or singly ionized alkaline earth atom). The frequency $\omega$ of the radiation is tuned near the $S_{1 / 2}, m_{J}=-\frac{1}{2} \leftrightarrow P_{3 / 2}$, $m_{J}=-\frac{3}{2}$ transition frequency $\omega_{0}$. The atom cycles nearly continuously between these levels since the dipole selection rules allow spontaneous decay only to the original ground level. A steady stream of fluorescence photons, which are readily detected, is emitted by the atom during this period. However, if the radiation is linearly polarized with the polarization direction perpendicular to the direction of the magnetic field, the $S_{1 / 2} \quad m_{J}=-\frac{1}{2} \leftrightarrow P_{3 / 2}$, $m_{J}=\frac{1}{2}$ transition is also allowed, although it is far from resonance. This transition is indicated by the dashed arrow in Fig. 1. A spontaneous decay from the upper $m_{J}=\frac{1}{2}$ level can then leave the atom in the $m_{J}=\frac{1}{2}$ ground level. This spontaneous Raman transition into the $m_{J}=\frac{1}{2}$ ground level takes the atom out of the $m_{J}=-\frac{1}{2} \leftrightarrow m_{J}=-\frac{3}{2}$ cycling loop, causing the emitted fluorescence to suddenly stop. The off-resonant $S_{1 / 2}$, $m_{J}=+\frac{1}{2} \leftrightarrow P_{3 / 2}, m_{J}=-\frac{1}{2} \rightarrow S_{1 / 2}, m_{J}=-\frac{1}{2}$ spontaneous Raman transition (not shown in Fig. 1) will return the atom to the cycling loop where it will resume scattering.

In a previous paper, ${ }^{6}$ we calculated the behavior of this system when the magnetic field strength is large enough that $\alpha \gg \gamma, \Omega, \Delta$, where $\hbar \alpha / 2$ is the energy separation between adjacent excited-state sublevels, $3 \hbar \alpha / 4$ is the separation between the ground-state sublevels, $\gamma$ is the excited-state spontaneous decay rate, $\Omega$ is the on-resonance Rabi frequency for the $S_{1 / 2}, m_{J}=-\frac{1}{2} \rightarrow P_{3 / 2}, m_{J}=-\frac{3}{2}$ transition, and $\Delta=\omega-\omega_{0}$ (see Fig. 1).

We have used a single ${ }^{24} \mathrm{Mg}^{+}$ion confined by the static magnetic and electric fields of a Penning trap ${ }^{7}$ to demonstrate this process. The magnetic field for the data presented here was $1.39 \mathrm{~T}$ which gives $a=(2 \pi) 52$ $\mathrm{GHz}=1200 \gamma$, where $\gamma=(2 \pi) 43 \mathrm{MHz}$. Therefore, the condition $\alpha \gg \gamma, \Omega, \Delta$ is quite readily achieved in our experiment.

For the trap used in this work (Fig. 2), we achieved a large collection solid angle ( $>1 \%$ of $4 \pi \mathrm{sr}$ ), compared to an earlier apparatus, ${ }^{8}$ by splitting the ring electrode into two halves in the plane perpendicular to the magnetic field direction. ${ }^{9}$ The relative trap dimensions were chosen, with the aid of a computer, to make the potential nearly quadratic at the trap center. ${ }^{10}$ The pressure of back- 


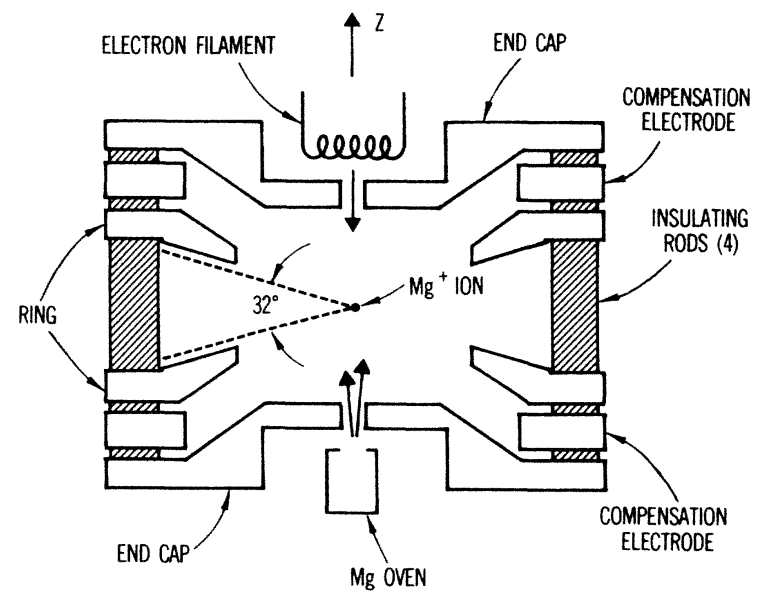

FIG. 2. Cross-sectional drawing of the Penning trap used in the experiment. The shaded regions are ceramic support rods. The end-cap separation is $1.38 \mathrm{~cm}$ and the inner diameter of the ring electrode is $1.63 \mathrm{~cm}$. The two ring electrodes and the two compensation electrodes were grounded and the two end caps were at $+12 \mathrm{~V}$ for this experiment.

ground gas in the apparatus was less than $10^{-8} \mathrm{~Pa}$.

The $3 S_{1 / 2}$ to $3 P_{3 / 2}$ transition wavelength for $\mathrm{Mg}^{+}$is $280 \mathrm{~nm}$. We generated up to $200 \mu \mathrm{W}$ of $280-\mathrm{nm}$ radiation by frequency doubling the output of a single-mode, continuous-wave dye laser in a deuterated ammonium dihydrogen-phosphate crystal. The frequency width of this radiation was much less than the natural width $\gamma$ of the transition. The laser beam was focused near the center of the trap with a beam waist $w_{0}=45 \mu \mathrm{m}$. The ions were laser cooled when $\Delta<0$, and since the beam was directed at an angle of $74^{\circ}$ relative to the magnetic field axis all of the ion's degrees of motion were cooled directly. The equilibrium temperature of the ion was only a fraction of $1 \mathrm{~K}$ when $\Delta \simeq-\gamma / 2$. $^{11}$ Under these conditions, the Doppler broadening of the transition frequency was much less than $\gamma$, as verified by a scan of the laser frequency, and the ion was confined to dimensions which were much less than $w_{0}$. In order to laser cool in a Penning trap, it is necessary to position the center of the laser beam radially outward from the trap axis. ${ }^{11}$ With a displacement of approximately $20 \mu \mathrm{m}$ and our value of $w_{0}$, the saturation parameter $\Omega^{2} / \gamma^{2}$ was equal to 1 at the position of the ion for a laser power of approximately $50 \mu \mathrm{W}$. From the definitions of Ref. 6, the ion spends twice as much time in the ground $m_{J}=-\frac{1}{2}$ sublevel as in the excited $m_{J}=-\frac{3}{2}$ level when $\Omega=\gamma$ and $\Delta=0$. The fluorescence photons were collected in a direction perpendicular to both the magnetic field and laser beam directions by focusing onto a photomultiplier tube. The maximum detected photon count rate was $2 \times 10^{5} / \mathrm{s}$ for a single ion.

Data exhibiting quantum jumps are displayed in the inset in Fig. 3. The horizontal axis is divided into time intervals of $0.5 \mathrm{~ms}$ duration. The vertical axis indicates the number of photons counted during each time interval and the points representing each interval are connected by straight lines. The sudden changes in fluorescence level are due to quantum jumps between the ground-state sub-

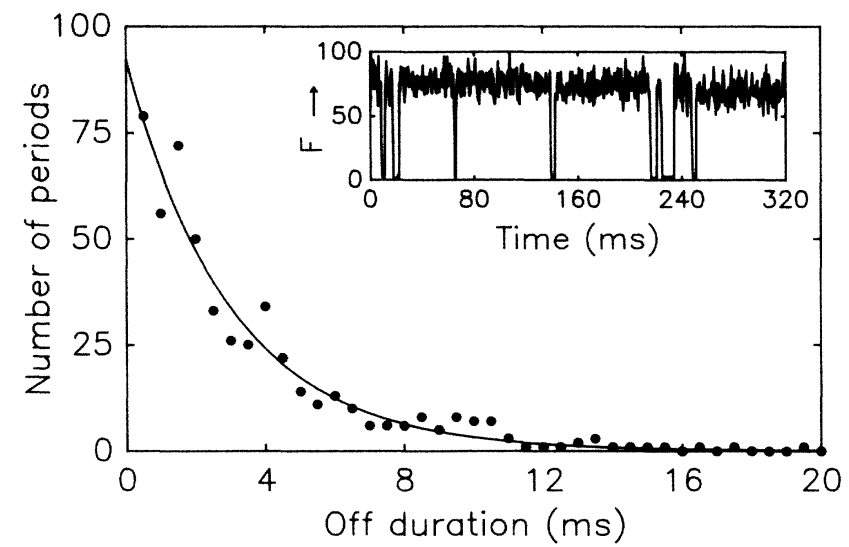

FIG. 3. A histogram of the duration of fluorescence-off periods for a data run. The number of off periods which have a particular duration are plotted vs the duration. This data run had 512 off periods in $20 \mathrm{~s}$. The solid line is a fit of the data to Eq. (2). Inset: A portion of a data run showing quantum jumps in fluorescence $F$ as a function of time.

levels via spontaneous Raman transitions.

For other realizations of quantum jumps, the dynamics of the population evolution and, therefore, the statistics of the emitted fluorescence are governed by effective twostate rate equations. ${ }^{12}$ That is, there are rates $R_{+}$and $R$ - for the atom to make transitions out of or into the energy levels which comprise the strongly fluorescing or "on" system and the system of levels which comprise the "off" system. $\left(R_{+}\right)^{-1}$ and $\left(R_{-}\right)^{-1}$ are equal to the mean duration of the fluorescence on periods, $\left\langle T_{\text {on }}\right\rangle$, and off periods, $\left\langle T_{\text {off }}\right\rangle$, respectively. Although the system considered here is substantially different than those investigated before, it was shown in Ref. 6 that the dynamics of this system should also be governed by effective two-state rate equations. The expressions for the rates $R_{-}$and $R_{+}$,

$$
\begin{aligned}
& R_{+}=\left\langle T_{\text {on }}\right\rangle^{-1}=\Omega^{2} \gamma /\left(18 \alpha^{2}\right), \\
& R_{-}=\left\langle T_{\text {off }}\right\rangle^{-1}=8 \Omega^{2} \gamma /\left(9 \alpha^{2}\right),
\end{aligned}
$$

are simple because of the off-resonance nature of the excitation, in contrast with the previously considered systems. ${ }^{12}$

A consequence of this type of dynamics is that the distribution of fluorescence-on and fluorescence-off times should be exponential. In Fig. 3, we plot the duration of fluorescence-off periods versus the number of periods in the data run which are off for this duration. The solid line is a fit to the equation

$$
W_{\text {off }}(t)=\left\langle T_{\text {off }}\right\rangle^{-1} \exp \left(-t /\left\langle T_{\text {off }}\right\rangle\right) .
$$

The data are consistent with a distribution of this form.

An important prediction of Ref. 6 is that the ratio of the mean duration of the fluorescence-on periods to the mean duration of the fluorescence-off periods should be a value which is only very weakly dependent on the variable parameters of the system, such as laser power and frequency. This is in contrast with the rather strong dependence of this ratio on system parameters in the other experi- 
TABLE I. A summary of the data for seven runs with different values of the saturation parameter $\Omega^{2} / \gamma^{2}$. Also shown are the calculated results when the stimulated Raman coherences between excited states are either included or neglected. Each data run contained 60000 time intervals whose length was either $0.25,0.33$, or $0.5 \mathrm{~ms}$. $\left\langle T_{\text {off }}\right\rangle$ ranged from $9.5 \mathrm{~ms}$ for run 1 to $2.3 \mathrm{~ms}$ for run 7 . Data runs in which the laser frequency varied excessively have been omitted from the table.

\begin{tabular}{|c|c|c|c|c|c|}
\hline Run & $\Omega^{2} / \gamma^{2}$ & $-\Delta / \gamma$ & $\frac{\left\langle T_{\text {on }}\right\rangle}{\left\langle T_{\text {off }}\right\rangle} \quad$ Calc & $\frac{\left\langle T_{\text {on }}\right\rangle}{\left\langle T_{\text {off }}\right\rangle}$ & $\begin{array}{c}\text { Measured } \\
\frac{\left\langle T_{\text {on }}\right\rangle}{\left\langle T_{\text {off }}\right\rangle}\end{array}$ \\
\hline 1 & $0.65 \pm 0.09$ & $0.67 \pm 0.03$ & $19.1 \pm 0.2$ & 16.09 & $15.76 \pm 0.84$ \\
\hline 2 & $1.82 \pm 0.16$ & $0.22 \pm 8.18$ & $25.8 \pm 0.5$ & 16.03 & $16.02 \pm 0.67$ \\
\hline 3 & $1.89 \pm 0.16$ & $0.20 \pm 8.20$ & $26.1 \pm 0.6$ & 16.03 & $15.52 \pm 0.58$ \\
\hline 4 & $2.02 \pm 0.16$ & $0.84 \pm 0.05$ & $21.7 \pm 0.3$ & 16.11 & $16.58 \pm 0.59$ \\
\hline 5 & $2.45 \pm 0.25$ & $1.07 \pm 0.05$ & $21.0 \pm 0.2$ & 16.13 & $16.13 \pm 0.70$ \\
\hline 6 & $2.61 \pm 0.20$ & $0.60 \pm 0.05$ & $24.4 \pm 0.6$ & 16.08 & $17.35 \pm 0.70$ \\
\hline 7 & $2.64 \pm 0.16$ & $0.50 \pm 0.06$ & $25.2 \pm 0.6$ & 16.07 & $16.26 \pm 0.69$ \\
\hline
\end{tabular}

ments. $^{1-4}$ Reference 6 shows that, for $\alpha \gg \gamma, \Omega, \Delta$,

$$
\left\langle T_{\text {on }}\right\rangle /\left\langle T_{\text {off }}\right\rangle=16+O(\Delta / \alpha) .
$$

We might intuitively expect that $\left\langle T_{\text {on }}\right\rangle /\left\langle T_{\text {off }}\right\rangle$ would increase by a factor of 2 when the $S_{1 / 2}, m_{J}=-\frac{1}{2} \rightarrow P_{3 / 2}$, $m_{J}=-\frac{3}{2}$ transition is saturated, since approximately half the population which was available in the $m_{J}=-\frac{1}{2}$ ground level to be transferred to the $m_{J}=\frac{1}{2}$ ground level is "stranded" in the excited state. However, the stimulated Raman coherence between the $m_{J}=-\frac{3}{2}$ and $m_{J}=\frac{1}{2}$ excited-state sublevels, through the $m_{J}=-\frac{1}{2}$ groundstate sublevel, compensates for the depletion of population in the ground state. ${ }^{6}$

We have investigated this phenomenon by measuring $\left\langle T_{\text {on }}\right\rangle /\left\langle T_{\text {off }}\right\rangle$ for various values of the saturation parameter $\Omega^{2} / \gamma^{2}$. Table I shows the results of analyzing seven different data runs with saturation parameters ranging between 0.65 and 2.64. The value of the saturation parameter and its uncertainty were obtained by fitting the distribution of fluorescence-off time durations to Eq. (2) to find $\left\langle T_{\text {off }}\right\rangle$ and its uncertainty. Equation (1) then relates $\left\langle T_{\text {off }}\right\rangle$ to $\Omega$. This procedure gives results which are consistent with estimates of the expected saturation parameter from independent measurements of the laser power and the laser beam waist $w_{0}$. The rate of fluorescence emitted by the ion during the fluorescence-on periods depends on the average population in the exited $m_{J}=-\frac{3}{2}$ level, which depends on the detuning $\Delta .^{6}$ Therefore, if the count rate for $\Delta=0$ is known, then $\Delta$ for each data run is determined by the mean detected count rate for the fluorescence-on periods of that run. The count rate for $\Delta=0$ is found from the maximum detected rate when the laser is scanned through the resonance. ${ }^{13,14}$ The uncertainty given for $\Delta$ in Table $I$ is due to the uncertainty in the count rate for $\Delta=0$. The fourth column gives the expected $\left\langle T_{\text {on }}\right\rangle /\left\langle T_{\text {off }}\right\rangle$ ratio if the coherences between excited states are neglected. ${ }^{6}$ The fifth column is the expected value of the ratio when the coherences are included in the calculation. Since $\Delta \neq 0$, there is a small departure from the value of 16. The uncertainty in this calculated ratio is small compared to the uncertainty in the measured value of $\left\langle T_{\text {on }}\right\rangle /\left\langle T_{\text {off }}\right\rangle$ and is therefore not reported. Finally, the last column is our measured value for $\left\langle T_{\text {on }}\right\rangle /\left\langle T_{\text {off }}\right\rangle$ for each run. This quantity was extracted from the data by fitting the distribution of detected counts per time interval for each run to a sum of two Gaussian curves, one corresponding to the fluorescence-off periods and the other to the fluorescence-on periods. The ratio $\left\langle T_{\text {on }}\right\rangle /\left\langle T_{\text {off }}\right\rangle$ is then equal to the ratio of the areas under the Gaussian curves. The uncertainty given is estimated from the uncertainty in determining the fitting parameters. The mean for the seven runs is 16.23 and the sample standard deviation is 0.60. Combining the seven runs (weighted equally) gives $\left\langle T_{\text {on }}\right\rangle /\left\langle T_{\text {off }}\right\rangle=16.23 \pm 0.26$. A comparison of columns 5 and 6 reveals that our data are quite consistent with the result of the coherent theory, which for the given set of values of $\Delta$ gives a mean value for the ratio of 16.08 .

In this experiment, spontaneous Raman transitions induced by a single laser are responsible for quantum jumps into and out of the long-lived "shelving" level." The shelving level is a ground-state sublevel rather than an electronic excited metastable state as in the previous demonstrations. The properties of this level structure have allowed the theory of quantum jumps, in which the dynamics of the jumps are described by effective two-level rate equations, to be tested with high precision. This experiment is a realization of "single-atom optical pumping." Ordinarily, the effects of optical pumping are evident only in the time-averaged values of the level populations. However, in single-atom optical pumping, the dynamical evolution of the level populations is revealed by quantum jumps in the fluorescence intensity. Finally, the existence of stimulated Raman coherences between excited states has been shown to play an important role in both the population dynamics and in the steady-state values of the level populations.

The authors thank C. Weimer for help in constructing parts of the apparatus and E. C. Beaty for performing computer calculations for the trap design. We also thank L. Hollberg and F. Diedrich for helpful comments on the manuscript. The authors gratefully acknowledge the support of the Air Force Office of Scientific Research and the Office of Naval Research. One of us (R.G.H.) thanks the National Research Council for support. 
*Present address: Physics Department, Rice University, Houston, TX 77251.

${ }^{1}$ W. Nagourney, J. Sandberg, and H. Dehmelt, Phys. Rev. Lett. 56, 2797 (1986).

${ }^{2}$ J. C. Bergquist, R. G. Hulet, W. M. Itano, and D. J. Wineland, Phys. Rev. Lett. 57, 1699 (1986).

${ }^{3}$ Th. Sauter, W. Neuhauser, R. Blatt, and P. E. Toschek, Phys. Rev. Lett. 57, 1696 (1986).

${ }^{4}$ M. A. Finn, G. W. Greenlees, and D. A. Lewis, Opt. Commun. 60, 149 (1986).

${ }^{5}$ R. G. Hulet and D. J. Wineland, Bull. Am. Phys. Soc. 31, 938 (1986).

${ }^{6}$ R. G. Hulet and D. J. Wineland, Phys. Rev. A 36, 2758 (1987).

${ }^{7}$ See, for example, H. G. Dehmelt, Adv. At. Mol. Phys. 3, 53
(1967); 5, 109 (1969); D. J. Wineland, W. M. Itano, and R. S. Van Dyck, Jr., ibid. 19, 135 (1983).

${ }^{8}$ D. J. Wineland and W. M. Itano, Phys. Lett. 82A, 75 (1981).

${ }^{9}$ M. H. Prior and H. A. Shugart, Phys. Rev. Lett. 27, 902 (1971).

${ }^{10}$ E. C. Beaty, J. Appl. Phys. 61, 2118 (1987).

${ }^{11}$ W. M. Itano and D. J. Wineland, Phys. Rev. A 25, 35 (1982).

${ }^{12}$ H. J. Kimble, R. J. Cook, and A. L. Wells, Phys. Rev. A 34, 3190 (1986), and references therein.

${ }^{13}$ R. E. Drullinger, D. J. Wineland, and J. C. Bergquist, Appl. Phys. 22, 365 (1980).

${ }^{14}$ W. Nagourney, G. Janik, and H. Dehmelt, Proc. Natl. Acad. Sci. U.S.A. 80, 643 (1983). 